\title{
On the cardinal utility equivalence of biseparable preferences
}

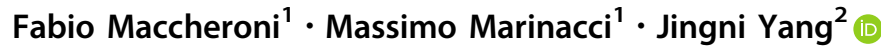

Accepted: 23 January 2022 / Published online: 18 February 2022

(C) The Author(s) 2022

\begin{abstract}
We establish a simple condition, based on the willingness to bet on events, under which two biseparable preferences have cardinally equivalent utilities

Keywords Biseparable preferences · Cardinal equivalent utilities · Willingness to bet
\end{abstract}

\section{Introduction and preliminaries}

Ghirardato and Marinacci (2002) argued that when comparing the ambiguity attitudes of two decision makers (DMs) with biseparable preferences, it is important to keep the cardinal utilities equivalent to factor out risk attitudes that, otherwise, would intrude in the comparison and confound it. With this motivation, they establish the desired cardinal utility equivalence via a trade-off condition. In this work, we present a simpler condition, based on the willingness to bet on events, that ensures this equivalence (Theorem 6).

We consider a state space $S$, finite or infinite, endowed with an event algebra $\Sigma$, and a connected topological space $X$ of consequences. We denote by $\mathcal{F}$ the collection of (simple) acts, i.e., of the $\Sigma$-measurable maps from $S$ to $X$ that assume finitely many values. ${ }^{1}$

The DM's preference on $\mathcal{F}$ is modeled by a binary relation $\succsim$, with symmetric part $\sim$ and asymmetric part $\succ$. As usual, consequences are identified with constant acts and so, with a standard abuse of notation, $\succsim$ ranks also consequences. In

\footnotetext{
1 Everything we write also applies to the case in which preferences are defined on a subset of $\mathcal{F}$ which contains all binary acts.
}

We dedicate this paper, with admiration, to Peter Wakker on the occasion of his 65th birthday. We thank Han Bleichrodt for useful comments.

\footnotetext{
$\triangle$ Jingni Yang

jingni.yang@anu.edu.au

1 Università Bocconi and IGIER, Milan, Italy

2 Australian National University, Canberra, Australia
} 
particular, throughout, we assume that the preference $\succsim$ is non-trivial, i.e., there exist two consequences $x$ and $y$ with $x \succ y$.

Given any two consequences $x$ and $y$, with $x \succsim y$, we denote by $x A y$ the binary act that yields $x$ if event $A$ occurs and $y$ otherwise, interpreted as a bet on event $A$. An event $A$ is essential if there exist two consequences $x \succsim y$ such that

$$
x \succ x A y \succ y .
$$

A functional $V: \mathcal{F} \rightarrow \mathbb{R}$ represents $\succsim$ if, for all $f, g \in \mathcal{F}$,

$$
f \succsim g \Longleftrightarrow V(f) \geq V(g) \text {. }
$$

The preference $\succsim$ is monotone if, given any two acts $f$ and $g, f(s) \succsim g(s)$ for all $s$ in $S$ implies $f \succsim g$. A functional $V$ represents a monotone preference $\succsim$ if and only if it is itself monotone: given any two acts $f$ and $g, f(s) \succsim g(s)$ for all $s$ in $S$ implies $V(f) \geq V(g)$.

\section{Biseparable preferences}

Ghirardato and Marinacci (2001) introduced the following general class of preferences. $^{2}$

Definition 1 A monotone preference $\succsim$ on $\mathcal{F}$ is biseparable if there exist a functional $V: \mathcal{F} \rightarrow \mathbb{R}$ that represents $\succsim$ and functions $\rho=\rho_{V}: \Sigma \rightarrow \mathbb{R}$ and $u=$ $u_{V}: X \rightarrow \mathbb{R}$ such that

$$
V(x A y)=u(x) \rho(A)+u(y)(1-\rho(A))
$$

for all consequences $x \succsim y$ and all events $A$. The functional $V$ is called a canonical representation of $\succsim$.

It is immediate to see that

$$
u(x)=V(x) \quad \forall x \in X
$$

and that, given any $x \succ y$ in $X$,

$$
\rho(A)=\frac{V(x A y)-V(y)}{V(x)-V(y)} \quad \forall A \in \Sigma .
$$

The functions $u$ and $\rho$ are thus unique given $V$. We call $\rho$ a canonical willingness to bet of the DM since $x A y \succsim x B y$ if and only if $\rho(A) \geq \rho(B)$ and we call $u$ a canonical utility function since it represents $\succsim$ on $X{ }^{3}$ The monotonicity of $\succsim$ implies that $\rho$ is a capacity, ${ }^{4}$ while its non-triviality implies that $u$ is non-constant.

\footnotetext{
2 It includes Choquet expected utility (Schmeidler, 1989), maxmin expected utility (Gilboa \& Schmeidler, 1989) and $\alpha$-maxmin expected utility (Ghirardato et al., 2004). See Gilboa (2009), Wakker (2010) and Gilboa and Marinacci (2013) for background material on these models.

3 On this terminology, see Ghirardato and Marinacci (2001) p. 868.

${ }^{4}$ That is, a set function such that $0=\rho(\emptyset) \leq \rho(A) \leq \rho(B) \leq \rho(S)=1$ for all events $A \subseteq B$.
} 
In reading the next definition, recall that a preference $\succsim$ is (topologically) continuous on $X$ if the sets $\{y \in X: y \succsim x\}$ and $\{y \in X: x \succsim y\}$ are closed for all $x$ in $X$.

Definition 2 A biseparable preference $\succsim$ is solvable if it is continuous on $X$ and if each bet has a certainty equivalent.

The next preliminary result characterizes solvable biseparable preferences through the continuity of canonical utility functions. ${ }^{5}$

Proposition 3 Let $\succsim$ be a biseparable preference on $\mathcal{F}$ that admits an essential event. The following conditions are equivalent:

(i) $\succsim$ is solvable;

(ii) any canonical utility function is continuous;

(iii) some canonical utility function is continuous;

(iv) $\succsim$ is continuous on $X$ and each act has a certainty equivalent.

Proof (i) implies (ii). Since $\succsim$ is continuous, by Lemma 8 , there exists a strictly increasing function $f: \operatorname{Im} u \rightarrow \mathbb{R}$ such that $\tilde{u}=f \circ u$ is continuous. Therefore, $\operatorname{Im} \tilde{u}$ is an interval because $X$ is connected. Let $A$ be an essential event, with $\alpha=\rho(A) \in(0,1)$. Since each bet has a certainty equivalent, for each $v_{1} \geq v_{2}$ in $\operatorname{Im} u$, with $u\left(x_{1}\right)=v_{1}$ and $u\left(x_{2}\right)=v_{2}$, we have $V\left(x_{1} A x_{2}\right)=\alpha v_{1}+(1-\alpha) v_{2}=u(x)$ for some $x \in X$. Therefore, $\alpha v_{1}+(1-\alpha) v_{2} \in \operatorname{Im} u$. By Corollary 14 (in the special case when the topological vector space is the real line), this implies that $\overline{\operatorname{Im} u}$ is an interval. We have $\operatorname{Im} f=\{(f \circ u)(x): x \in X\}=\operatorname{Im} \tilde{u}$ and so $\operatorname{Im} f$ is an interval. By Proposition 10, $f$ is monotonically continuous. Since $f$ is strictly increasing, by Corollary 11 , the inverse $f^{-1}$ is continuous. Therefore, also $u=f^{-1} \circ \tilde{u}$ is continuous.

(iii) implies (iv). Suppose that $u$ is continuous. By Lemma $8, \succsim$ is continuous on $X$. It remains to show that each act has a certainty equivalent. For any act $f$, denote by $x^{f}$ and $x_{f}$ a best and a worst consequence of $f$. Monotonicity implies that $x^{f} \succsim f \succsim x_{f}$. Then,

$$
u\left(x^{f}\right)=V\left(x^{f}\right) \geq V(f) \geq V\left(x_{f}\right)=u\left(x_{f}\right) .
$$

The image $\operatorname{Im} u$ of $u$ is an interval because $X$ is connected. Therefore, $V(f) \in$ $\left[u\left(x_{f}\right), u\left(x^{f}\right)\right]$ implies that there exists $z \in X$ such that $z \sim f$, as desired. This completes the proof as it is obvious that (ii) implies (iii) and that (iv) implies (i). $\square$

\section{Cardinal equivalence}

In this section, we compare preferences over acts that are possibly defined on different state spaces. To this end, for $i=1,2$ we denote by $\Sigma_{i}$ an event algebra defined on a state space $S_{i}$, with typical element $A_{i}$, and by $\succsim_{i}$ a preference defined over the corresponding collection $\mathcal{F}_{i}$ of acts $f: S_{i} \rightarrow X$.

\footnotetext{
5 Ghirardato and Marinacci (2001) p. 874 axiomatize biseparable preferences that feature a continuous $u$.
} 
The following definition introduces a comparative notion for betting behavior.

Definition 4 Preference $\succsim_{1}$ is more willing to bet on event $A_{1}$ than preference $\succsim_{2}$ on an event $A_{2}$ if, for all consequences $x, y, z \in X$ with $x \succsim_{1} y$ and $x \succsim_{2} y$,

$$
x A_{2} y \succ_{2} z \Longrightarrow x A_{1} y \succ_{1} z \text {. }
$$

In words, whenever $\mathrm{DM}_{2}$ prefers to bet on event $A_{2}$ than to get a sure alternative, so does $\mathrm{DM}_{1}$ for a bet on event $A_{1}$. For biseparable preferences, this comparative notion has the following implication that corroborates the interpretation of $\rho$ in terms of willingness to bet.

Lemma 5 For $i=1,2$, let $\succsim_{i}$ be a solvable biseparable preference with a canonical representation $V_{i}$ and an essential event $A_{i}$. If $\succsim_{1}$ is more willing to bet on $A_{1}$ than $\succsim_{2}$ is on $A_{2}$, then $\rho_{1}\left(A_{1}\right) \geq \rho_{2}\left(A_{2}\right)$.

Proof To ease notation, set $\rho_{1}=\rho_{1}\left(A_{1}\right)$ and $\rho_{2}=\rho_{2}\left(A_{2}\right)$. By essentiality, we have $\rho_{1}, \rho_{2} \in(0,1)$. We want to show that $\rho_{1} \geq \rho_{2}$. Assume, per contra, that $\rho_{1}<\rho_{2}$. First observe that, by taking $y=x$ (so that automatically $x \succsim_{1} y$ and $x \succsim_{2} y$ ) and using the contrapositive of (4), it holds

$$
z{ }_{1} x \Rightarrow z{ }_{2} x
$$

for all $x, z \in X$. Thus,

$$
u_{1}(z) \geq u_{1}(x) \Rightarrow z \succsim_{1} x \Rightarrow z \succsim_{2} x \Rightarrow u_{2}(z) \geq u_{2}(x) .
$$

To ease notation, set $U_{1}=\operatorname{Im} u_{1}$ and $U_{2}=\operatorname{Im} u_{2}$. Since $X$ is connected and the nonconstant functions $u_{1}, u_{2}: X \rightarrow \mathbb{R}$ are continuous, both $U_{1}$ and $U_{2}$ are non-degenerate intervals. By (5), there exists an increasing and continuous (by Corollary 12) map $\varphi: U_{1} \rightarrow U_{2}$ such that $u_{2}=\varphi \circ u_{1}$. Let $a<b$ in $U_{1}$ and set $c=\rho_{1} b+\left(1-\rho_{1}\right) a$. Take $x, y, z \in X$ such that $b=u_{1}(x), c=u_{1}(z)$ and $a=u_{1}(y)$. Since $a<b$, we have $x \succsim_{1} y$ and so $x \succsim_{2} y$. We thus have

$$
\begin{aligned}
c=\rho_{1} b+\left(1-\rho_{1}\right) a & \Rightarrow u_{1}(z)=\rho_{1} u_{1}(x)+\left(1-\rho_{1}\right) u_{1}(y) \\
& \Rightarrow z \sim{ }_{1} x A_{1} y \Longrightarrow z \succsim{ }_{2} x A_{2} y \\
& \Rightarrow u_{2}(z) \geq \rho_{2} u_{2}(x)+\left(1-\rho_{2}\right) u_{2}(y) \\
& \Rightarrow \varphi(c) \geq \rho_{2} \varphi(b)+\left(1-\rho_{2}\right) \varphi(a) \\
& \Rightarrow \varphi(c) \geq \rho_{1} \varphi(b)+\left(1-\rho_{1}\right) \varphi(a),
\end{aligned}
$$

where the last implication holds because $\varphi$ is increasing and $\rho_{2}>\rho_{1}$. Thus, for all $a<b$ in $U_{1}$,

$$
c=\rho_{1} b+\left(1-\rho_{1}\right) a \Rightarrow \varphi(c) \geq \rho_{1} \varphi(b)+\left(1-\rho_{1}\right) \varphi(a) .
$$

This implies that $\varphi$ is concave (see, e.g., Hardy et al., 1934, p. 73).

Let $\lambda=\rho_{1}\left(1-\rho_{2}\right) / \rho_{2}\left(1-\rho_{1}\right)$. Since $0<\rho_{1}<\rho_{2}<1$, we have $\lambda \in(0,1)$. By (6), we have 


$$
b-c=b-\left(\rho_{1} b+\left(1-\rho_{1}\right) a\right)=\left(1-\rho_{1}\right)(b-a)>0
$$

and, by the preceding calculations,

$$
-\varphi(c) \leq-\left(\rho_{2} \varphi(b)+\left(1-\rho_{2}\right) \varphi(a)\right)
$$

as well as

$$
c-a=\rho_{1} b+\left(1-\rho_{1}\right) a-a=\rho_{1}(b-a)>0
$$

and

$$
\varphi(c)-\varphi(a) \geq \rho_{2} \varphi(b)+\left(1-\rho_{2}\right) \varphi(a)-\varphi(a)=\rho_{2}(\varphi(b)-\varphi(a)) .
$$

Then,

$$
\frac{\varphi(b)-\varphi(c)}{b-c} \leq \frac{\varphi(b)-\left(\rho_{2} \varphi(b)+\left(1-\rho_{2}\right) \varphi(a)\right)}{\left(1-\rho_{1}\right)(b-a)}=\frac{1-\rho_{2}}{1-\rho_{1}} \frac{\varphi(b)-\varphi(a)}{b-a}
$$

and

$$
\frac{\varphi(c)-\varphi(a)}{c-a} \geq \frac{\left(\rho_{2} \varphi(b)+\left(1-\rho_{2}\right) \varphi(a)\right)-\varphi(a)}{\rho_{1}(b-a)}=\frac{\rho_{2}}{\rho_{1}} \frac{\varphi(b)-\varphi(a)}{b-a} .
$$

By multiplying both sides of the latter inequality by $\rho_{1} / \rho_{2}$,

$$
\frac{\varphi(b)-\varphi(a)}{b-a} \leq \frac{\rho_{1}}{\rho_{2}} \frac{\varphi(c)-\varphi(a)}{c-a} .
$$

Thus,

$$
\frac{\varphi(b)-\varphi(c)}{b-c} \leq \frac{1-\rho_{2}}{1-\rho_{1}} \frac{\varphi(b)-\varphi(a)}{b-a} \leq \frac{1-\rho_{2}}{1-\rho_{1}} \frac{\rho_{1}}{\rho_{2}} \frac{\varphi(c)-\varphi(a)}{c-a}=\lambda \frac{\varphi(c)-\varphi(a)}{c-a} .
$$

Summing up, for all $a<b$ in $U_{1}$,

$$
c=\rho_{1} b+\left(1-\rho_{1}\right) a \Longrightarrow \frac{\varphi(b)-\varphi(c)}{b-c} \leq \lambda \frac{\varphi(c)-\varphi(a)}{c-a} .
$$

Now, fix $c \in$ int $U_{1}$. Take a sequence $a_{n} \uparrow c$ and a sequence $b_{n} \downarrow c$ such that $a_{n}<b_{n}$ in $U_{1}$ and

$$
c=\rho_{1} b_{n}+\left(1-\rho_{1}\right) a_{n}
$$

for all $n \geq 1$. For example, for $n$ large enough,

$$
c=\rho_{1} \underbrace{\left(c+\frac{1}{n \rho_{1}}\right)}_{b_{n}}+\left(1-\rho_{1}\right) \underbrace{\left(c-\frac{1}{n\left(1-\rho_{1}\right)}\right)}_{a_{n}} .
$$

By (7), for each $n$, we have 


$$
\frac{\varphi\left(b_{n}\right)-\varphi(c)}{b_{n}-c} \leq \lambda \frac{\varphi(c)-\varphi\left(a_{n}\right)}{c-a_{n}} .
$$

Since $\varphi$ is concave, we can take the limits as $n \rightarrow \infty$, thus getting

$$
\varphi_{+}^{\prime}(c) \leq \lambda \varphi_{-}^{\prime}(c) .
$$

Since $\lambda \in(0,1)$, if $\varphi$ is differentiable at $c \in$ int $U_{1}$, it holds $\varphi^{\prime}(c)=0$. Therefore, $\varphi^{\prime}$ is zero on the differentiability set $D_{1} \subseteq$ int $U_{1}$ on which $\varphi_{+}^{\prime}=\varphi_{-}^{\prime}=\varphi^{\prime}$. Since the complement in int $U_{1}$ of the set $D_{1}$ is at most countable, the monotonicity of $\varphi_{+}^{\prime}$ implies that $\varphi_{+}^{\prime}$ is zero on int $U_{1}$. Therefore, $\varphi$ is constant on int $U_{1}$. As $\varphi$ is continuous, it is then constant on the entire interval $U_{1}$. Hence, $u_{2}$ is constant too, a contradiction. We conclude that $\rho_{1} \geq \rho_{2}$.

In particular, with a common state space $S$ and event algebra $\Sigma$, if $\succsim_{1}$ is more willing to bet than $\succsim_{2}$ on all events, then this lemma implies that $\rho_{1} \geq \rho_{2}$ on $\Sigma$. Wang (2019) proves this "global" result in an Anscombe-Aumann setting.

Next, we present our main result. It provides a simple condition-the existence of two essential events, one per DM, for which the DMs share the same willingness to bet-under which biseparable preferences have cardinally equivalent utilities.

Theorem 6 For $i=1,2$, let $\succsim_{i}$ be a solvable biseparable preference with a canonical representation $V_{i}$ and an essential event $A_{i}$. The following conditions are equivalent:

(i) $\succsim_{1}$ is as willing to bet on event $A_{1}$ as $\succsim_{2}$ on event $A_{2}$.

(ii) $\succsim_{1}$ and $\succsim_{2}$ have cardinally equivalent utilities $u_{1}$ and $u_{2},{ }^{6}$ and $\rho_{1}\left(A_{1}\right)=\rho_{2}\left(A_{2}\right)$.

Proof We maintain the conventions introduced in the proof of Lemma 5, and we only prove that (i) implies (ii), the converse being trivial. First, observe that $\succsim_{1}$ and $\succsim_{2}$ coincide on $X$ and that, by Lemma 5, we have $\rho_{1}\left(A_{1}\right)=\rho_{2}\left(A_{2}\right)$. Since both $u_{1}$ and $u_{2}$ are continuous, there exists a strictly increasing and continuous (by Corollary 12) map $\varphi: U_{1} \rightarrow U_{2}$ such that $u_{2}=\varphi \circ u_{1}$ and $u_{1}=\varphi^{-1} \circ u_{2}$. For all $a<b$ in $U_{1}$, it thus holds (6) and so $\varphi$ is concave. A similar argument proves that $\varphi^{-1}$ is concave. The strictly increasing map $\varphi$ is thus affine. Therefore, $u_{1}$ and $u_{2}$ are cardinally equivalent.

Summing up, this theorem shows - using concavity arguments - that a simple behavioral condition characterizes the cardinal equivalence of the utility functions of two biseparable preferences. This significantly simplifies the condition used by Ghirardato and Marinacci (2002) that requires the two preferences to be ordinally equivalent (i.e., to agree on $X$ ) and cardinally symmetric, a trade-off assumption that permits to use techniques from additive conjoint measurement (cf. Wakker, 1989).

\footnotetext{
6 That is, $u_{1}$ is a positive affine transformation of $u_{2}$ (so, $u_{1}$ and $u_{2}$ are equal up to a common normalization).
} 
A remarkable consequence of the last result is the cardinal equivalence of canonical representations, so their uniqueness up to a common normalization, and the full-fledged uniqueness of the willingness to bet.

Corollary 7 For $i=1,2$, let $V_{i}$ be any two canonical representations of a solvable biseparable preference $\succsim$ that admits an essential event. Then, $V_{1}$ is cardinally equivalent to $V_{2}$ and $\rho_{1}=\rho_{2}$.

Thus, a solvable biseparable preference features a canonical trio $(V, u, \rho)$, in which the canonical representation $V$ and the canonical utility $u$ are cardinally unique, while the willingness to bet $\rho$ is unique. This trio is the representation skeleton of the preference that, as argued in Ghirardato et al. (2005), is able to separate utility and beliefs.

Proof By Theorem 6, $u_{1}$ is cardinally equivalent to $u_{2}$, that is, there exist $m>0$ and $q \in \mathbb{R}$ such that $u_{2}(z)=m u_{1}(z)+q$ for all $z \in X$. Let $f \in \mathcal{F}$. By Proposition 3 , there is $x_{f} \in X$ with $f \sim x_{f}$. Hence, $V_{1}(f)=u_{1}\left(x_{f}\right)$ and $V_{2}(f)=u_{2}\left(x_{f}\right)$, so that

$$
V_{2}(f)=u_{2}\left(x_{f}\right)=m u_{1}\left(x_{f}\right)+q=m V_{1}(f)+q
$$

Thus, $V_{2}=m V_{1}+q$. Let $x \succ y$ in $X$. By (3), for each event $A$, we have

$$
\rho_{2}(A)=\frac{V_{2}(x A y)-V_{2}(y)}{V_{2}(x)-V_{2}(y)}=\frac{m V_{1}(x A y)+q-m V_{1}(y)-q}{m V_{1}(x)+q-m V_{1}(y)-q}=\rho_{1}(A) .
$$

Thus, $\rho_{1}=\rho_{2}$.

\section{A Appendix: background material}

In this appendix, we present some mathematical results that our analysis requires. For the sake of completeness, in the next two subsections, we carry out an analysis which is actually more general than what strictly needed for this paper. Before doing this, we report a decision-theoretic result that we use in the paper [cf. Theorem 3.5 of Fishburn, 1970].

Lemma 8 Let $\succsim$ be a preference on a connected topological space X that admits a utility function $u: X \rightarrow \mathbb{R}$. The following conditions are equivalent:

(i) $\succsim$ is topologically continuous;

(ii) there exists a strictly increasing transformation $f: \operatorname{Im} u \rightarrow \mathbb{R}$ such that $\tilde{u}=$ $f \circ u$ is continuous.

\section{A.1 An intermediate value theorem on a non-convex domain}

Say that a function $f: A \rightarrow \mathbb{R}$ defined on a subset $A$ of the real line is monotonically continuous if, for any monotone sequences $\left\{x_{n}\right\}$ and $\left\{y_{n}\right\}$ in $A$, it holds 


$$
x_{n} \leq x_{n+1} \leq y_{n+1} \leq y_{n} \quad \text { and } \quad y_{n}-x_{n} \rightarrow 0 \Longrightarrow f\left(y_{n}\right)-f\left(x_{n}\right) \rightarrow 0 .
$$

An important example of monotonically continuous functions are uniformly continuous functions. On interval domains, continuous functions as well are monotonically continuous, as next we show.

Lemma 9 A continuous function $f: A \rightarrow \mathbb{R}$ is monotonically continuous provided $A$ is an interval.

Proof Let $f: A \rightarrow \mathbb{R}$ be continuous, and let $\left\{x_{n}\right\}$ and $\left\{y_{n}\right\}$ be two monotone sequences in $A$ satisfying $x_{n} \leq x_{n+1} \leq y_{n+1} \leq y_{n}$ and $y_{n}-x_{n} \rightarrow 0$. Since $A$ is an interval, and $\left[x_{n}, y_{n}\right]$ is a decreasing sequence of compact sets with vanishing diameter, there exists $z \in A$ such that $x_{n} \rightarrow z$ and $y_{n} \rightarrow z$. By the continuity of $f$, we then have $f\left(x_{n}\right) \rightarrow f(z)$ and $f\left(y_{n}\right) \rightarrow f(z)$, which in turn implies that $f\left(y_{n}\right)-f\left(x_{n}\right) \rightarrow 0$.

The next result is, essentially, an intermediate value theorem on a non-convex domain.

Proposition 10 Let $f: A \rightarrow \mathbb{R}$ be a monotone function defined on a subset of the real line whose closure $\bar{A}$ is an interval. The following conditions are equivalent:

(i) $f$ is monotonically continuous;

(ii) $\overline{\operatorname{Im} f}$ is an interval;

(iii) $f$ has a unique continuous extension on the interval $A \cup \operatorname{int} \bar{A}$.

The set $A \cup$ int $\bar{A}$ consists of the interior points of $\bar{A}$, along with the endpoints of $\bar{A}$ that belong to $A$. The natural $\operatorname{logarithm} \log x$, with $A=(0, \infty)$ and $A \cup \operatorname{int} \bar{A}=A$, is a simple example showing that in (iii) we cannot replace $A \cup$ int $\bar{A}$ with $\bar{A}$.

To see that continuity in (i) is not enough, note that the monotone and continuous function $f: \mathbb{Q} \rightarrow \mathbb{R}$ given by

$$
f(x)=\left\{\begin{array}{cc}
1 & \text { if } x>\sqrt{2} \\
-1 & \text { if } x<\sqrt{2}
\end{array}\right.
$$

is not monotonically continuous and has a discrete closed image $\operatorname{Im} f=\{1,-1\} .^{7}$

Proof of Proposition 10 We show that

$$
\text { (i) } \Rightarrow(\text { iii }) \Rightarrow(\text { ii }) \Rightarrow(\text { i) }
$$

We assume that $A$ is nonsingleton, otherwise the result is trivial, and that $f$ is increasing (the decreasing case follows by changing the sign of $f$ ). To ease notation, in the proof, we set $I=A \cup$ int $\bar{A}$.

(i) implies (iii). For all $z \in \bar{A}$, set

\footnotetext{
7 By adding to it the identity function, the function $f$ can be made strictly monotone.
} 


$$
f(z)=\sup \{f(x): x \in A \text { and } x \leq z\} \quad \text { and } \quad \bar{f}(z)=\inf \{f(y): y \in A \text { and } z \leq y\} .
$$

Clearly, $f \leq \bar{f}$. It is easy to check that that $f$ and $\bar{f}$ are the minimal and maximal increasing extensions of $f$ to $\bar{A}$, respectively. They are finite on $I$ but can be infinite on $\bar{A} \backslash I$, that is, on the endpoints of $\bar{A}$ that do not belong to $A$.

Let $z \in$ int $\bar{A}$ (which is nonempty because $\bar{A}$ is a nonsingleton interval). There exist $a<b$ such that $a<z<b$ and $[a, b] \subseteq \bar{A}$. Let $M \geq 1$ be large enough so that

$$
a<z-\frac{1}{M}<z+\frac{1}{M}<b .
$$

For all $m \geq M$,

$$
a<z-\frac{1}{m}<z-\frac{1}{m+1}<z<z+\frac{1}{m+1}<z+\frac{1}{m}<b
$$

and so the intervals

$$
\left(z-\frac{1}{m}, z-\frac{1}{m+1}\right) \text { and }\left(z+\frac{1}{m+1}, z+\frac{1}{m}\right)
$$

are disjoint and contained in $\bar{A}$. Then, there exist

$$
x_{m} \in\left(z-\frac{1}{m}, z-\frac{1}{m+1}\right) \cap A \quad \text { and } \quad y_{m} \in\left(z+\frac{1}{m+1}, z+\frac{1}{m}\right) \cap A .
$$

Clearly, $x_{m}<x_{m+1}<y_{m+1}<y_{m}$ and $y_{m}-x_{m} \rightarrow 0$. We actually have $x_{m} \uparrow z$ and $y_{m} \downarrow z$. By monotone continuity, $f\left(y_{m}\right)-f\left(x_{m}\right) \rightarrow 0$. Since, for all $m \geq M$, we have $f\left(x_{m}\right) \leq \sup \{f(x): x \in A$ and $x \leq z\}=f(z) \leq \bar{f}(z)=\inf \{f(y): y \in A$ and $z \leq y\} \leq f\left(y_{m}\right)$

passage to the limit, as $m \rightarrow \infty$, delivers $f(z)=\bar{f}(z) \in \mathbb{R}$. Being extensions of $f$ to $\bar{A}$, the functions $f$ and $\bar{f}$ also coincide and are finite on $A$. Thus, the function $\tilde{f}: I \rightarrow \mathbb{R}$ defined by

$$
\tilde{f}(z)=\underline{f}(z)=\bar{f}(z)
$$

is a monotone extension of $f$ to $I=A \cup$ int $\bar{A}$. Now, let $z \in$ int $\bar{A}$. For each $\varepsilon>0$, there exists $m_{\varepsilon} \geq M$ such that $\left|f\left(x_{m}\right)-f\left(y_{m}\right)\right|<\varepsilon$ for all $m \geq m_{\varepsilon}$. The set

$$
U_{\varepsilon}=\left(z-\frac{1}{m_{\varepsilon}+1}, z+\frac{1}{m_{\varepsilon}+1}\right)
$$

is a neighborhood of $z$ (in $\mathbb{R}$ and it is contained in $I$ ). For all $w \in U_{\varepsilon}$,

$$
f\left(x_{m_{\varepsilon}}\right) \leq \sup \{f(x): x \in A \text { and } x \leq w\}=\tilde{f}(w)=\inf \{f(y): y \in A \text { and } w \leq y\} \leq f\left(y_{m_{\varepsilon}}\right)
$$

but also 
$f\left(x_{m_{\varepsilon}}\right) \leq \sup \{f(x): x \in A$ and $x \leq z\}=\tilde{f}(z)=\inf \{f(y): y \in A$ and $z \leq y\} \leq f\left(y_{m_{\varepsilon}}\right)$

That is,

$$
|\tilde{f}(w)-\tilde{f}(z)| \leq\left|f\left(x_{m_{\varepsilon}}\right)-f\left(y_{m_{\varepsilon}}\right)\right|<\varepsilon .
$$

This shows the continuity of $\tilde{f}$ on int $\bar{A}$.

Let $z \in A \backslash$ int $\bar{A} \subseteq \bar{A} \backslash$ int $\bar{A}$. Then $z \in \partial \bar{A}$ and there are two possibilities:

- if $z=\min \bar{A}$, define $x_{m}=z$ and $y_{m}$ as above to prove the continuity of $\tilde{f}$ at $z$;

- if $z=\max \bar{A}$, define $x_{m}$ as above $y_{m}=z$ to prove the continuity of $\tilde{f}$ at $z$.

Summing up, $\tilde{f}$ is a continuous extension of $f$ to $I$. It is the only continuous extension of $f$ to $I$ because of the density of $A$ in $\bar{A}$.

(iii) implies (ii). Let $\varphi$ be the continuous extension of $f$ on the interval $I=A \cup$ int $\bar{A}$. Since $\operatorname{Im} f \subseteq \operatorname{Im} \varphi$, we have $\overline{\operatorname{Im} f} \subseteq \overline{\operatorname{Im} \varphi}$. Let $r \in \operatorname{Im} \varphi$. There exists $x \in I$ such that $\varphi(x)=r$, as well as a sequence $\left\{x_{n}\right\} \subseteq A$ such that $x_{n} \rightarrow x$ (because $I \subseteq \bar{A})$. Then, $f\left(x_{n}\right)=\varphi\left(x_{n}\right) \rightarrow \varphi(x)=r$. Therefore, $r \in \overline{\operatorname{Im} f}$ and this proves that $\operatorname{Im} \varphi \subseteq \overline{\operatorname{Im} f}$. Therefore, $\overline{\operatorname{Im} \varphi} \subseteq \overline{\operatorname{Im} f}$. We conclude that $\overline{\operatorname{Im} f}=\overline{\operatorname{Im} \varphi}$. Hence, $\overline{\operatorname{Im} f}$ is an interval because $\operatorname{Im} \varphi$ is an interval and so does $\overline{\operatorname{Im} \varphi}$.

(ii) implies (i). Assume per contra that there are two monotone sequences $\left\{x_{n}\right\}$ and $\left\{y_{n}\right\}$ satisfying $x_{n} \leq x_{n+1} \leq y_{n+1} \leq y_{n}, y_{n}-x_{n} \rightarrow 0$ and $f\left(y_{n}\right)-f\left(x_{n}\right) \nrightarrow 0$. By the monotonicity of $f$, we have $f\left(x_{n}\right) \uparrow \underline{k} \in \overline{\operatorname{Im} f}$ and $f\left(y_{n}\right) \downarrow \bar{k} \in \overline{\operatorname{Im} f}$. Thus, $f\left(y_{n}\right)-f\left(x_{n}\right) \rightarrow \bar{k}-\underline{k}>0$. Since the closure of $\operatorname{Im} f$ is an interval containing $(\underline{k}, \bar{k})$, there exist $x^{*}, y^{*} \in A$ such that $\underline{k}<f\left(x^{*}\right)<f\left(y^{*}\right)<\bar{k}$. It follows that

$$
f\left(x_{n}\right)<f\left(x^{*}\right)<f\left(y^{*}\right)<f\left(y_{n}\right)
$$

for all $n$. The monotonicity of $f$ implies that $x_{n}<x^{*}<y^{*}<y_{n}$ for all $n$. Thus, $y_{n}-$ $x_{n}>y^{*}-x^{*}>0$ for all $n$, which contradicts $y_{n}-x_{n} \rightarrow 0$. We conclude that $f$ satisfies (8) and so is monotonically continuous.

The fact that (i) implies (iii) means, inter alia, that monotone continuity is a stronger property than continuity, unless $A$ is an interval (Lemma 9). This, and more, is the content of the next result.

Corollary 11 Let $f: A \rightarrow \mathbb{R}$ be a monotone function defined on a subset of the real line whose closure $\bar{A}$ is an interval. If $: A \rightarrow \mathbb{R}$ is monotonically continuous, then it is continuous. If, in addition, $f$ is strictly monotone, then also the inverse $f^{-1}$ : $\operatorname{Im} f \rightarrow A$ is continuous.

Proof Let $f$ be strictly increasing (for the decreasing case is then enough to consider $-f$ ). If we prove that its continuous extension $\varphi$ to the interval $I=A \cup \operatorname{int} \bar{A}$ is strictly increasing, it follows that $\varphi: I \rightarrow \operatorname{Im} \varphi$ is continuous and bijective. Then, 
$\varphi^{-1}: \operatorname{Im} \varphi \rightarrow I$ is continuous and bijective. Therefore, $f^{-1}: \operatorname{Im} f \rightarrow A$ is continuous because $f^{-1}$ is the restriction of $\varphi^{-1}$ to $\operatorname{Im} f .^{8}$

We thus show that $\varphi$ is strictly increasing if $f$ is. Let $z<w$ in $I$. Since $[z, w] \subset$ $I \subseteq \bar{A}$ is an interval with non-empty interior, a construction similar to the one we carried out in the proof of Proposition 10 shows that there exist two monotone sequences $\left\{y_{n}\right\}$ and $\left\{v_{n}\right\}$ in $A$ such that $y_{m} \downarrow z$ and $v_{n} \uparrow w$. Then, there exists $N \geq 1$ such that, for all $n \geq N, y_{n}<y_{N}<v_{N}<v_{n}$. Thus,

$$
\varphi\left(y_{n}\right)=f\left(y_{n}\right)<f\left(y_{N}\right)<f\left(v_{N}\right)<f\left(v_{n}\right)=\varphi\left(v_{n}\right) .
$$

Passing to the limits, thanks to the continuity of $\varphi$ we get

$$
\varphi(z)=\lim _{n} f\left(y_{n}\right) \leq f\left(y_{N}\right)<f\left(v_{N}\right) \leq \lim _{n} f\left(v_{n}\right)=\varphi(w) .
$$

That is, $\varphi(z)<\varphi(w)$. We conclude that $\varphi$ is strictly increasing.

To see that the continuity of the inverse is a non-trivial property, consider the strictly monotone function $f:[0,1) \cup[2,3) \rightarrow \mathbb{R}$ given by

$$
f(x)= \begin{cases}x & \text { if } x \in[0,1) \\ x-1 & \text { if } x \in[2,3)\end{cases}
$$

This function is continuous, so monotonically continuous because Lemma continues to hold when $A$ is the union of intervals with pairwise disjoint closures. The image $\operatorname{Im} f$ is an interval, but the inverse $f^{-1}$ is obviously not continuous. The closure $\bar{A}=[0,1] \cup[2,3]$ is not an interval.

Proposition 10 has, as a further consequence, a well-known calculus version of the monotone intermediate value theorem, which we report just because we need to refer to it in the paper.

Corollary 12 Let $A$ be an interval of the real line. A monotone function $f: A \rightarrow \mathbb{R}$ is continuous if and only if $\operatorname{Im} f$ is an interval.

Summing up, monotone continuity emerges as a property that makes it possible to prove intermediate value theorems on non-convex domains.

\section{A.2 Convexity}

Proposition 13 A closed set $C$ in a topological vector space $V$ is convex if and only if, for each $x, y \in C$, there exists $\alpha_{x, y} \in(0,1)$ such that $\alpha_{x, y} x+\left(1-\alpha_{x, y}\right) y \in C$.

Proof We prove the "if" since the converse trivially holds. Assume per contra that $C$ is not convex. Then, there exist $x, y \in C$ and $\beta_{x, y} \in(0,1)$ such that

$$
\beta_{x, y} x+\left(1-\beta_{x, y}\right) y \notin C .
$$

Define $f:[0,1] \rightarrow V$ by $f(\alpha)=\alpha x+(1-\alpha) y$. The function $f$ is continuous because

\footnotetext{
${ }^{8}$ Let $t \in \operatorname{Im} f \subseteq \operatorname{Im} \varphi$, then there exists $x \in A$ such that $t=f(x)=\varphi(x)$. Since $\varphi$ is injective, we have that $x=f^{-1}(t)=\varphi^{-1}(t)$.
} 
the operations of addition and scalar multiplication are continuous. Since $C$ is closed in $V$, then $f^{-1}(C)$ is closed in $[0,1]$. In turn, this implies that the sets of the real line

$$
L^{*}=f^{-1}(C) \cap\left[0, \beta_{x, y}\right]=\left\{\alpha \in[0,1]: \alpha x+(1-\alpha) y \in C \text { and } \alpha \leq \beta_{x, y}\right\}
$$

and

$$
L_{*}=f^{-1}(C) \cap\left[\beta_{x, y}, 1\right]=\left\{\alpha \in[0,1]: \alpha x+(1-\alpha) y \in C \text { and } \alpha \geq \beta_{x, y}\right\}
$$

are both compact. Thus, there is a maximum $a \in L^{*}$ and a minimum $b \in L_{*}$. Since $\beta_{x, y} \notin L^{*} \cup L_{*}$, we have

$$
0 \leq a<\beta_{x, y}<b \leq 1
$$

Set $z_{a}=f(a)=a x+(1-a) y$ and $z_{b}=f(b)=b x+(1-b) y$. Since $a \in L^{*}$ and $b \in L_{*}$, then $z_{a}$ and $z_{b}$ belong to $C$. It follows that there exists $\alpha=\alpha_{x, y} \in(0,1)$ such that

$$
\alpha z_{a}+(1-\alpha) z_{b} \in C
$$

We then have

$$
\alpha(a x+(1-a) y)+(1-\alpha)(b x+(1-b) y) \in C
$$

that is,

$$
(\alpha a+(1-\alpha) b) x+(1-(\alpha a+(1-\alpha) b)) y \in C .
$$

Now, $a<\alpha a+(1-\alpha) b<b$. If $\alpha a+(1-\alpha) b \leq \beta_{x, y}$, the maximality of $a$ in $L^{*}$ is contradicted, while if $\alpha a+(1-\alpha) b \geq \beta_{x, y}$ it is the minimality of $b$ in $L_{*}$ to be contradicted. In any case, an absurd conclusion follows.

Corollary 14 The closure $\bar{C}$ of a set $C$ in a topological vector space $V$ is convex if and only if there exists $\alpha \in(0,1)$ such that, for each $x, y \in C, \alpha x+(1-\alpha) y \in \bar{C}$.

Proof The "only if" holds because $C \subseteq \bar{C}$ and the set $\bar{C}$ is convex. As to the converse, assume that $\alpha C+(1-\alpha) C \subseteq C$ for some $\alpha \in(0,1)$. In view of Proposition, it is enough to show that, for each $x, y \in \bar{C}, \alpha x+(1-\alpha) y \in \bar{C}$, i.e., that $\alpha \bar{C}+(1-\alpha) \bar{C} \subseteq \bar{C}$. Following Pettis (1956) p. 235, observe that

$$
\alpha \bar{C}+(1-\alpha) \bar{C} \subseteq \overline{\alpha C+(1-\alpha) C} \subseteq \bar{C}
$$

as desired.

Funding Open Access funding enabled and organized by CAUL and its Member Institutions.

Open Access This article is licensed under a Creative Commons Attribution 4.0 International License, which permits use, sharing, adaptation, distribution and reproduction in any medium or format, as long as you give appropriate credit to the original author(s) and the source, provide a link to the Creative Commons licence, and indicate if changes were made. The images or other third party material in this article are included in the article's Creative Commons licence, unless indicated otherwise in a credit line to the material. If material is not included in the article's Creative Commons licence and your intended 
use is not permitted by statutory regulation or exceeds the permitted use, you will need to obtain permission directly from the copyright holder. To view a copy of this licence, visit http:// creativecommons.org/licenses/by/4.0/.

\section{References}

Fishburn, P. C. (1970). Utility Theory for Decision Making. Wiley.

Ghirardato, P., Maccheroni, F., \& Marinacci, M. (2004). Differentiating ambiguity and ambiguity attitude. Journal of Economic Theory, 118, 133-173.

Ghirardato, P., Maccheroni, F., \& Marinacci, M. (2005). Certainty independence and the separation of utility and beliefs. Journal of Economic Theory, 120, 129-136.

Ghirardato, P., \& Marinacci, M. (2001). Risk, ambiguity, and the separation of utility and beliefs. Mathematics of Operations Research, 26, 864-890.

Ghirardato, P., \& Marinacci, M. (2002). Ambiguity made precise: A comparative foundation. Journal of Economic Theory, 102, 251-289.

Gilboa, I. (2009). Theory of Decision Under Uncertainty. Cambridge University Press.

Gilboa, I., \& Marinacci, M. (2013). Ambiguity and the Bayesian paradigm. In D. Acemoglu, M. Arellano, \& E. Dekel (Eds.), Advances in Economics and Econometrics: Theory and Applications. Cambridge University Press.

Gilboa, I., \& Schmeidler, D. (1989). Maxmin expected utility with non-unique prior. Journal of Mathematical Economics, 18, 141-153.

Hardy, G. H., Littlewood, J. E., \& Pólya, G. (1934). Inequalities. Cambridge University Press.

Pettis, B. J. (1956). Separation theorems for convex sets. Mathematics Magazine, 29, 233-247.

Schmeidler, D. (1989). Subjective probability and expected utility without additivity. Econometrica, 57, 571-587.

Wakker, P. (1989). Additive Representations of Preferences: A New Foundation of Decision Analysis. Dordrecht: Kluwer.

Wakker, P. (2010). Prospect Theory. Cambridge University Press.

Wang, F. (2019). Comparative ambiguity attitudes, mimeo.

Publisher's Note Springer Nature remains neutral with regard to jurisdictional claims in published maps and institutional affiliations. 